\title{
Response to Combined Peptide Receptor Radionuclide Therapy and Checkpoint Immunotherapy with Ipilimumab Plus Nivolumab in Metastatic Merkel Cell Carcinoma
}

\author{
Justin Ferdinandus ${ }^{1}$, Wolfgang P. Fendler ${ }^{1}$, Katharina Lueckerath ${ }^{1}$, Christoph Berliner ${ }^{1}$, Sabine Kurzidem ${ }^{2}$, \\ Eva Hadaschik ${ }^{2}$, Joachim Klode ${ }^{2}$, Lisa Zimmer ${ }^{2}$, Elisabeth Livingstone ${ }^{2}$, Dirk Schadendorf ${ }^{2}$, Ken Herrmann ${ }^{1}$, \\ Juergen C. Becker, ${ }^{2,3}$, and Selma Ugurel ${ }^{2}$ \\ ${ }^{I}$ Department of Nuclear Medicine, University of Duisburg-Essen, and German Cancer Consortium, University Hospital Essen, Essen, \\ Germany; ${ }^{2}$ Department of Dermatology, University of Duisburg-Essen, and German Cancer Consortium, University Hospital Essen, \\ Essen, Germany; and ${ }^{3}$ Translational Skin Cancer Research, University Medicine Essen, Essen, Germany
}

\begin{abstract}
For patients with Merkel cell carcinoma (MCC) who are refractory to immune checkpoint inhibition (ICl), treatment options are limited. Few cases of MCCs have been reported to show responses to peptide receptor radionuclide therapy (PRRT). A combination of PRRT and ICl has not been reported in MCC to date. A patient with metastatic MCC, who was resistant to first-line avelumab and acquired resistance to ipilimumab/nivolumab (IPI/NIVO) with additional radiotherapy, presented with multiple distant metastases. After confirmation of SSTR expression, treatment was continued with an additional 4 doses of IPI/NIVO combined with 2 cycles of PRRT. Treatment was well tolerated, with transient hemotoxicity and mild nausea. Restaging after 3 mo demonstrated an exceptional response. This case demonstrates the feasibility of combined treatment with IPI/NIVO and PRRT as an option for MCC patients progressing under ICl. Prospective evidence confirming the additive value of combining $\mathrm{ICl}$ and radionuclide therapy in a larger cohort is needed.
\end{abstract}

Key Words: immunotherapy; PRRT, SSTR; Merkel cell carcinoma; PD-L1

J Nucl Med 2022; 63:396-398

DOI: 10.2967/jnumed.121.262344

$\mathbf{M}$

erkel cell carcinoma (MCC) is an aggressive form of neuroendocrine skin cancer. In the United States, the incidence of MCC is around 0.8 per 100,000 , and the numbers continue to rise. Metastatic MCC (mMCC) is characterized by a particularly poor prognosis, with a 5-y overall survival rate of approximately $30 \%$ (1).

On the basis of the high immunogenicity of MCC and the need for more effective therapies, immune checkpoint inhibition (ICI) was successfully introduced for first- and second-line treatment of mMCC (2). Similar to many other neuroendocrine malignancies, MCC expresses somatostatin receptors (SSTRs) enabling receptortargeted PET and peptide receptor radionuclide therapy (PRRT) (3).

PRRT led to significantly prolonged survival compared with treatment with somatostatin analogs in patients with neuroendocrine

Received Mar. 22, 2021; revision accepted Jun. 14, 2021.

For correspondence or reprints, contact Justin Ferdinandus (justin. ferdinandus@uk-essen.de).

Published online Sep. 2, 2021.

COPYRIGHT @ 2022 by the Society of Nuclear Medicine and Molecular Imaging. tumors of the midgut (4). PRRT for mMCC exhibited a promising response in a few case reports (5-7). Here, we report the case of a patient with mMCC who progressed on multiple lines of ICI and subsequently responded well to combined therapy with ipilimumab plus nivolumab ICI and somatostatin PRRT.

\section{MATERIALS AND METHODS}

A 60-y-old, immune-competent man presented with a red lump on his right upper thigh. Surgical excision and histopathology revealed a high-grade MCC with positive margins. Sentinel lymph node biopsy confirmed nodal involvement. The tumor was MCPyV-positive, with a low tumor mutational burden, but lacked significant programmed death-ligand 1 expression.

The initial TNM stage was $\mathrm{pT} 2 \mathrm{pN} 1 \mathrm{~b} \mathrm{cM} 0$. Subsequently, the patient received adjuvant radiotherapy to the tumor bed $(60 \mathrm{~Gy})$ and the draining lymph node region (50 Gy). Follow-up CT staging demonstrated multiple enlarged retroperitoneal lymph nodes, indicative of metastases (Fig. 1). Systemic treatment with avelumab, $800 \mathrm{mg}$ every $2 \mathrm{wk}$, was initiated and tolerated well, but imaging after 4 doses revealed progressive disease (Fig. 1). The immunotherapeutic regimen was switched to ipilimumab, $3 \mathrm{mg} / \mathrm{kg}$, and nivolumab, $1 \mathrm{mg} / \mathrm{kg}$ every $3 \mathrm{wk}$ (IPI/NIVO), which was accompanied by severe diarrhea requiring treatment interruption. Additionally, radiotherapy (50.4 Gy) of the retroperitoneal lymph nodes was performed and resulted in a partial response (Fig. 1). At the same time, the patient took artesunate supplements $(8)$ and received multiple administrations of Newcastle disease virus; both treatments were given on the patient's own initiative outside our center (9).

Shortly after pausing immunotherapy, the patient developed oligoprogression with new bone metastases and started supportive treatment with denosumab and palliative radiation therapy delivered to selected bone metastases of the spine. After recovering from the previous immune-related toxicity, the patient started on nivolumab, $480 \mathrm{mg}$ every 4 wk as maintenance therapy, but again progressed dramatically within $30 \mathrm{~d}$, with several new skeletal lesions.

Restaging with SSTR PET confirmed high levels of SSTR expression at all sites of disease (bone and lymph nodes). We therefore decided to rechallenge the patient with IPI/NIVO together with PRRT. Written informed consent for publication was obtained from the patient.

\section{RESULTS}

The patient received a cumulative activity of $14.8 \mathrm{GBq}$ of ${ }^{177} \mathrm{Lu}$-DOTATOC over 2 cycles of PRRT (4). Posttreatment scintigraphy was performed to verify retention of the radionuclide in 


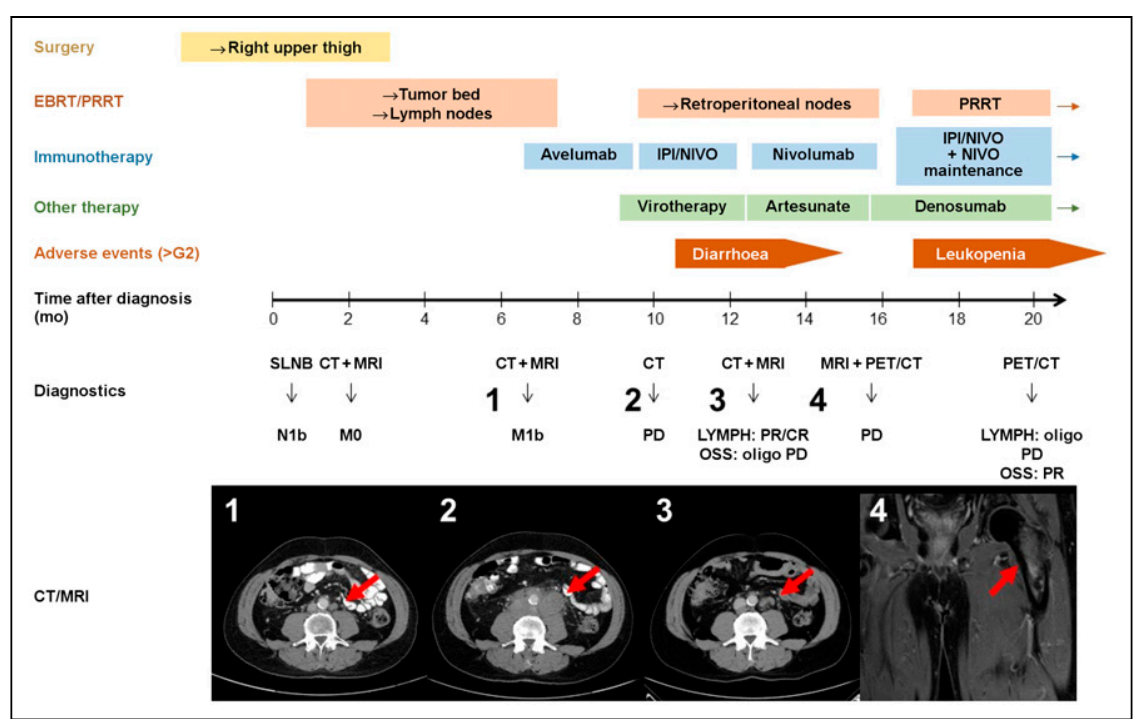

FIGURE 1. Retroperitoneal lymph node metastasis: flowchart of important events since initial diagnosis, and CT and MR images of retroperitoneal lymph node metastases (1-3; arrows). (1) After detection of lymph node metastasis, immunotherapy with avelumab was initiated. (2) After $1 \mathrm{mo}$, rapid increase in lactate dehydrogenase serum levels was noted, and restaging revealed progressive disease. Treatment was switched to IPI/NIVO plus radiotherapy to retroperitoneal lymph nodes. (3 and 4) Although lymph node metastases showed strong response to IPI/NIVO (3), new bone metastases were detected (4; arrow). $\mathrm{CR}=$ complete response; EBRT = external-beam radiation therapy; LYMPH = lymph node metastasis; OSS = bone metastasis; oligo PD = oligoprogressive disease; $\mathrm{PD}$ = progressive disease; $\mathrm{PR}=$ partial response.
Radiation leads to immunogenic cell death and activation of immune effector cells, which could enhance the efficacy of ICI therapy (11). Further, radiation alters the microenvironment of tumors via the expression of chemokines and release of antigens and neoantigens, allowing for immune cell infiltration of irradiated tissue (12). Conversely, ICI therapy may enhance the therapeutic effects of radiation by counteracting tumor hypoxia and influx of myeloid-derived suppressor cells, which are known mechanisms of radioresistance (13).

In contrast to external-beam radiation therapy, PRRT delivers radiation to every metastatic lesion with target expression. In the phase III NETTER-1 trial, compared with therapy with somatostatin analogs, PRRT led to prolonged progression-free survival and an improved quality of life in patients with midgut neuroendocrine tumors (4). Generally, PRRT is well tolerated, with lymphopenia, nausea, and fatigue as most frequent but often mild adverse effects (4). MCCs often express SSTR at moderate to high levels and are thus amenable to PRRT (14). In our patient, SSTR PET before treatment initiation revealed high and homoge-

tumor lesions (Fig. 2). IPI/NIVO was given as ipilimumab, $3 \mathrm{mg} / \mathrm{kg}$, and nivolumab, $1 \mathrm{mg} / \mathrm{kg}$, every $3 \mathrm{wk}$. The treatment was well tolerated, with transient grade 3 leukopenia, mild nausea (grade 2), and autoimmune-related thyroiditis (Common Terminology Criteria for Adverse Events grade 1). After 2 cycles of PRRT and 4 doses of IPI/NIVO, we performed restaging with SSTR PET. Here, we noted a marked response by multiple skeletal lesions (Fig. 2). However, we found one new lymph node metastasis on the left side of the neck, which was excised and histologically confirmed as MCC. Increased SSTR PET uptake in the thyroid was rated as thyroiditis. Because of his good treatment response on imaging and his good clinical condition, the patient currently continues PRRT at a $50 \%$ reduced dose (3.8 GBq/cycle), together with maintenance nivolumab, $480 \mathrm{mg}$ every $4 \mathrm{wk}$. At the time of submission, the patient had experienced an ongoing response for 5 mo.

\section{DISCUSSION}

Introduction of ICI greatly improved patient outcomes in advanced MCC (10). However, half the patients either primarily do not respond or exhibit acquired resistance. Our patient eventually progressed during all immunotherapeutic regimens but initially responded well to a combination ICI with IPI/NIVO. We therefore decided on an individual salvage regimen with reinduction of IPI/NIVO and addition of PRRT. neous expression of SSTR.

There is increasing interest in combining radionuclide therapy and ICI therapy. Promising preclinical data show that ICI enhances radionuclide therapy in prostate cancer (15), and 2 clinical trials are under way (NCT03658447 and NCT03805594). In a recent phase Ib trial in patients with neuroendocrine tumors of the lung, combined PRRT and nivolumab were well tolerated and showed signs of antitumor activity (16). Disease control was achieved in 3 of $9(33 \%)$ patients.

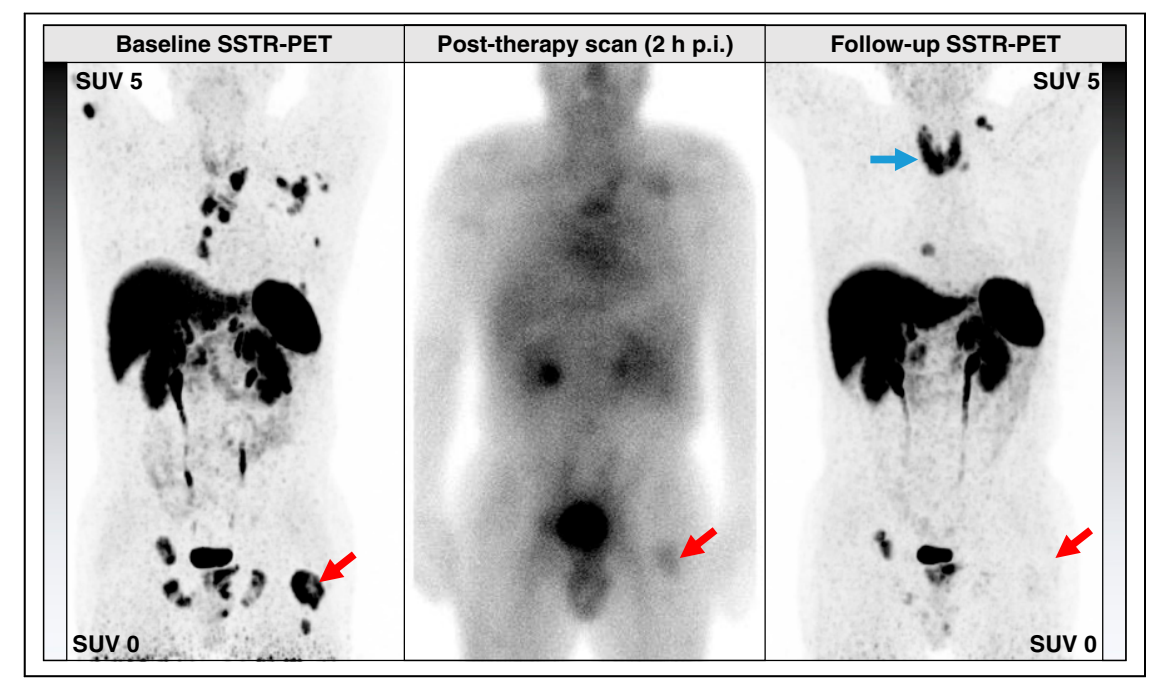

FIGURE 2. Response after IPI/NIVO plus PRRT: maximum-intensity projections of baseline and follow-up ${ }^{68} \mathrm{Ga}$-DOTATOC PET scans (SSTR PET) indicating favorable response of bone metastases (red arrows) to PRRT plus IPI/NIVO therapy. Physiologic uptake can be seen in liver, spleen, and urinary tract. Posttherapy scan after first PRRT cycle confirmed retention of ${ }^{177}$ Lu-DOTATOC in tumor sites. Follow-up SSTR PET $13 \mathrm{wk}$ after first cycle revealed new uptake in thyroid gland (blue arrow). Patient was diagnosed with autoimmune thyroiditis as side effect of ICl therapy with IPI/NIVO. 
The National Comprehensive Cancer Network guidelines have implemented the use of PRRT for several neuroendocrine tumors but not yet for MCC (17). To date, there have been only a few reported cases of successful PRRT in mMCC (5-7) and no cases of combined PRRT and immunotherapy. Recently, the GoTHAM trial (NCT04261855) was launched; it is investigating the efficacy of firstline avelumab plus PRRT for the treatment of mMCC. The 3-armed design compares avelumab, avelumab plus radiotherapy, or avelumab plus PRRT and stratifies patients by SSTR expression level. Results of this trial will shed light on the added value of radiation to ICI therapy. However, survival data are not expected before 2024. Trials evaluating PRRT as monotherapy in MCC are also urgently needed, ideally comparing PRRT to PRRT plus ICI. Meanwhile, PRRT alone or in combination with ICI therapy will be reserved for individuals who failed other therapeutic regimens. Our case underlines the intriguing promise of radionuclide therapy, potentially turning immunotherapyunresponsive into immunotherapy-responsive tumors.

\section{CONCLUSION}

We report the response to combined IPI/NIVO plus PRRT in a patient with $\mathrm{mMCC}$ refractory to multiple previous ICI regimens. Although a prospective clinical trial is under way, our case highlights the need for rapid clinical development of combination strategies for patients with no response to immunotherapy alone.

\section{DISCLOSURE}

Wolfgang Fendler is a consultant for BTG and Parexel/Calyx, and he received fees from RadioMedix, Bayer, and Parexel outside the submitted work. Lisa Zimmer reports honoraria from Roche, Bristol Myers Squibb, MSD, Novartis, and Pierre Fabre; has a consultancy or advisory role with Bristol Myers Squibb, Novartis, Pierre Fabre, Sun Pharma, Sanofi, and MSD; and receives research funding from Novartis and travel support from Bristol Myers Squibb, Pierre Fabre, Sanofi, Amgen, Novartis, and Sun Pharma. Elisabeth Livingstone served as a consultant for or has received honoraria from Amgen, Bristol-Myers Squibb, Merck Sharp \& Dohme, Novartis, Janssen, Medac, Sanofi, and Sunpharma and received travel support from Amgen, Merck Sharp \& Dohme, Bristol-Myers Squibb, Pierre Fabre, Sunpharma, and Novartis, outside the submitted work. Dirk Schadendorf acted as a speaker for, participated in an advisory board for, and received research funding or honoraria from 4SC Amgen, Array BioPharma, Bristol-Myers Squibb, Immunocore, Incyte, InFlarX, Helsinn, Merck Serono, Merck Sharp \& Dohme, Nektar, Neracare, Novartis, OncoSec, Pfizer, Philogen, Pierre Fabre, Regeneron Pharmaceuticals, Replimune, Roche, Sandoz-Hexal, Sanofi, Sun Pharma, and Ultimovacs. His group receives research grants from BristolMyers Squibb, Roche, Novartis, and Amgen. Ken Herrmann reports personal fees from Bayer, Sofie Biosciences, SIRTEX, Adacap, Curium, Endocyte, BTG, IPSEN, Siemens Healthineers, GE Healthcare, Amgen, Novartis, ymabs, HotKnot Therapeutics, Theragnostics, and Pharma15; other fees from Sofie Biosciences; nonfinancial support from ABX; and grants from BTG, outside the submitted work. Juergen Becker receives speakers' bureau honoraria from Amgen, Pfizer, MerckSerono, Recordati, and Sanofi and is a paid consultant/advisory board member/DSMB member for Boehringer Ingelheim, eTheRNA, InProTher, MerckSerono, Pfizer, 4SC, and Sanofi/Regeneron. His group receives research grants from Bristol-Myers Squibb, Merck Serono, HTG, IQVIA, and Alcedis. Selma Ugurel declares research support from Bristol Myers Squibb and Merck Serono; speakers' and advisory board honoraria from Bristol Myers Squibb, Merck Sharp \& Dohme, Merck Serono, Novartis, and Roche; and travel support from Bristol Myers Squibb and Merck Sharp \& Dohme. No other potential conflict of interest relevant to this article was reported.

\section{KEY POINTS}

QUESTION: Can immunotherapy and PRRT be combined to achieve a response in patients with $\mathrm{mMCC}$ who progressed under immunotherapy?

PERTINENT FINDINGS: Our case shows that combined immunotherapy and PRRT resulted in acceptable toxicity and an exceptional response.

IMPLICATIONS FOR PATIENT CARE: Combining immunotherapy and PRRT should be explored in prospective clinical trials; until then, it is a feasible option for patients who have exhausted all other options.

\section{REFERENCES}

1. Becker JC, Stang A, DeCaprio JA, et al. Merkel cell carcinoma. Nat Rev Dis Primers. 2017;3:17077.

2. Nghiem P, Bhatia S, Lipson EJ, et al. Durable tumor regression and overall survival in patients with advanced Merkel cell carcinoma receiving pembrolizumab as first-line therapy. J Clin Oncol. 2019;37:693-702.

3. Buder K, Lapa C, Kreissl MC, et al. Somatostatin receptor expression in Merkel cell carcinoma as target for molecular imaging. BMC Cancer. 2014;14:268.

4. Strosberg J, El-Haddad G, Wolin E, et al. Phase 3 trial of ${ }^{177}$ Lu-dotatate for midgut neuroendocrine tumors. $N$ Engl J Med. 2017;376:125-135.

5. Meier G, Waldherr C, Herrmann R, Maecke H, Mueller-Brand J, Pless M. Successful targeted radiotherapy with ${ }^{90} \mathrm{Y}$-DOTATOC in a patient with Merkel cell carcinoma. Oncology. 2004;66:160-163.

6. Kasi PM, Sharma A, Jain MK. Expanding the indication for novel theranostic ${ }^{177}$ Lu-dotatate peptide receptor radionuclide therapy: proof-of-concept of PRRT in Merkel cell cancer. Case Rep Oncol. 2019;12:98-103.

7. Moghadam SZ, Divband G, Shakeri S, Aryana K. Favorable response after only one cycle of peptide receptor radionuclide therapy with ${ }^{177}$ Lu-DOTATATE in a patient with metastatic Merkel cell carcinoma. Clin Nucl Med. 2019;44:650-652.

8. Sarma B, Willmes C, Angerer L, et al. Artesunate affects T antigen expression and survival of virus-positive Merkel cell carcinoma. Cancers (Basel). 2020;12:919.

9. Vasaturo A, Di Blasio S, Peeters DG, et al. Clinical implications of co-inhibitory molecule expression in the tumor microenvironment for DC vaccination: a game of stop and go. Front Immunol. 2013;4:417.

10. D'Angelo SP, Russell J, Lebbe C, et al. Efficacy and safety of first-line avelumab treatment in patients with stage IV metastatic Merkel cell carcinoma: a preplanned interim analysis of a clinical trial. JAMA Oncol. 2018;4:e180077.

11. Twyman-Saint Victor C, Rech AJ, Maity A, et al. Radiation and dual checkpoint blockade activate non-redundant immune mechanisms in cancer. Nature. 2015; 520:373-377.

12. Weichselbaum RR, Liang H, Deng L, Fu YX. Radiotherapy and immunotherapy: a beneficial liaison? Nat Rev Clin Oncol. 2017;14:365-379.

13. Wang Y, Liu ZG, Yuan H, et al. The reciprocity between radiotherapy and cancer immunotherapy. Clin Cancer Res. 2019;25:1709-1717.

14. Akaike T, Qazi J, Anderson A, et al. High somatostatin receptor expression and efficacy of somatostatin analogues in patients with metastatic Merkel cell carcinoma. Br J Dermatol. 2021;184:319-327.

15. Czernin J, Current K, Mona CE, et al. Immune-checkpoint blockade enhances ${ }^{225}$ Ac-PSMA617 efficacy in a mouse model of prostate cancer. J Nucl Med. 2021; 62:228-231.

16. Kim C, Liu SV, Subramaniam DS, et al. Phase I study of the ${ }^{177}$ Lu-DOTA ${ }^{0}-$ Tyr $^{3}-$ octreotate (Lutathera) in combination with nivolumab in patients with neuroendocrine tumors of the lung. J Immunother Cancer. 2020;8:e000980.

17. Shah MH, Goldner WS, Halfdanarson TR, et al. NCCN guidelines insights: neuroendocrine and adrenal tumors, version 2.2018. J Natl Compr Canc Netw. 2018;16:693-702. 